\title{
Scanning Transmission Electron Microscopy and Diffraction in SEM: Novel Approaches for In Situ Studies
}

\author{
Erdmann Spiecker, Christian Dolle, Peter Schweizer and Peter Denninger
}

Center for Nanoanalysis and Electron Microscopy (CENEM) \& Institute of Micro- and Nanostructure Research, Department of Materials Science and Engineering, Friedrich-AlexanderUniversität Erlangen-Nürnberg, 91058 Erlangen, Germany.

Current trends in electron microscopy point towards low-voltage applications. The reduction of beam energy offers several benefits like reduced beam damage and higher scattering cross sections, especially beneficial for the investigation of light elements. Widely recognized examples of light element-materials are graphene and carbon nanotubes, which have been thoroughly investigated by electron microscopy demanding primary beam energies below $80 \mathrm{keV}$ to exclude knock-on damage. While dedicated low-voltage TEMs are only slowly entering the commercial market, scanning electron microscopes (SEM) already use primary electron energies of 0.5-30 keV and thus offer an easy access to low-voltage microscopy.

The SEM is a highly versatile tool for the investigation of novel materials by offering a complex set of applicable techniques, less spatial limitations compared to TEM, high throughput and the possibility to use a wide range of available add-on tools (e.g. manipulators, gas injection systems, in situ equipment) while still maintaining resolution in the nm-range and high material contrast. Besides the well-established imaging of surfaces by secondary electrons and chemical contrast/composition by backscattered electrons and X-Ray spectroscopy, a SEM gives direct access to low-voltage scanning transmission electron microscopy (STEM). State-of-the-art segmented STEM detectors are very versatile and well suited for material and diffraction contrast imaging. However, a more quantitative interpretation of the STEM contrast (bright-field and dark-field) requires the knowledge of the electron diffraction pattern in the plane of the STEM-detector.

Here we report on a custom-built diffraction setup in the SEM enabling the simultaneous acquisition of real space and reciprocal space information in situ. Low Energy Nano Diffraction (LEND) in transmission is based on the combination of a fluorescent screen positioned below the sample with a dedicated CMOS camera. The technique has been implemented and successfully tested on graphene, silicon and polycrystalline gold. For graphene a hexagonal spot like diffraction pattern can be obtained due to the small convergence angle typically encountered in SEM [1]. The exemplary LEND pattern depicted in Fig. 2a has been taken from twisted bilayer graphene and allows for straightforward determination of the local twist angle (here $\sim 30^{\circ}$ ). By acquiring LEND patterns on a 2D array of sample positions the twisting angle can be mapped across large areas of twisted bilayer graphene. This is similar to 4D-STEM in TEM. However, with our setup we could already successfully demonstrate LEND down to electron energies of $0.5 \mathrm{keV}$ which opens up new opportunities in characterization of ultrathin low-Z materials.

The combination of STEM and LEND is furthermore important for reliable interpretation of STEM contrast in SEM since LEND allows to directly reveal the electron intensity that is collected by the different segments of the STEM detector. This enabled us to directly reveal basal plane dislocations in bilayer graphene and correctly interpret their contrast in dark-field STEM ([2], Fig. 2b). By combining this with the in situ capabilities of our SEM setup (Fig. 1) we could successfully manipulate the dislocations while simultaneously image them in dark-field STEM ([2], Fig. 2b, right). Further in situ STEM and LEND studies in SEM are ongoing. For instance, we recently installed a chipbased in situ heating device which we now use for investigation of phase transformation phenomena in thin films. [3] 


\section{References:}

[1] C Dolle, P Schweizer and E Spiecker, Proceedings of the Microscopy Conference MC2017, 21 25 August 2017, Lausanne (Switzerland), p. 498.

[2] P Schweizer, C Dolle and E Spiecker, Science Advances, accepted.

[3] The authors acknowledge funding from the German Research Foundation (DFG) through the Research Training Group GRK 1896 ,In situ microscopy with electrons, X-rays and scanning probes“ and the Collaborative Research Center SFB 953 „Synthetic carbon allotropes”.

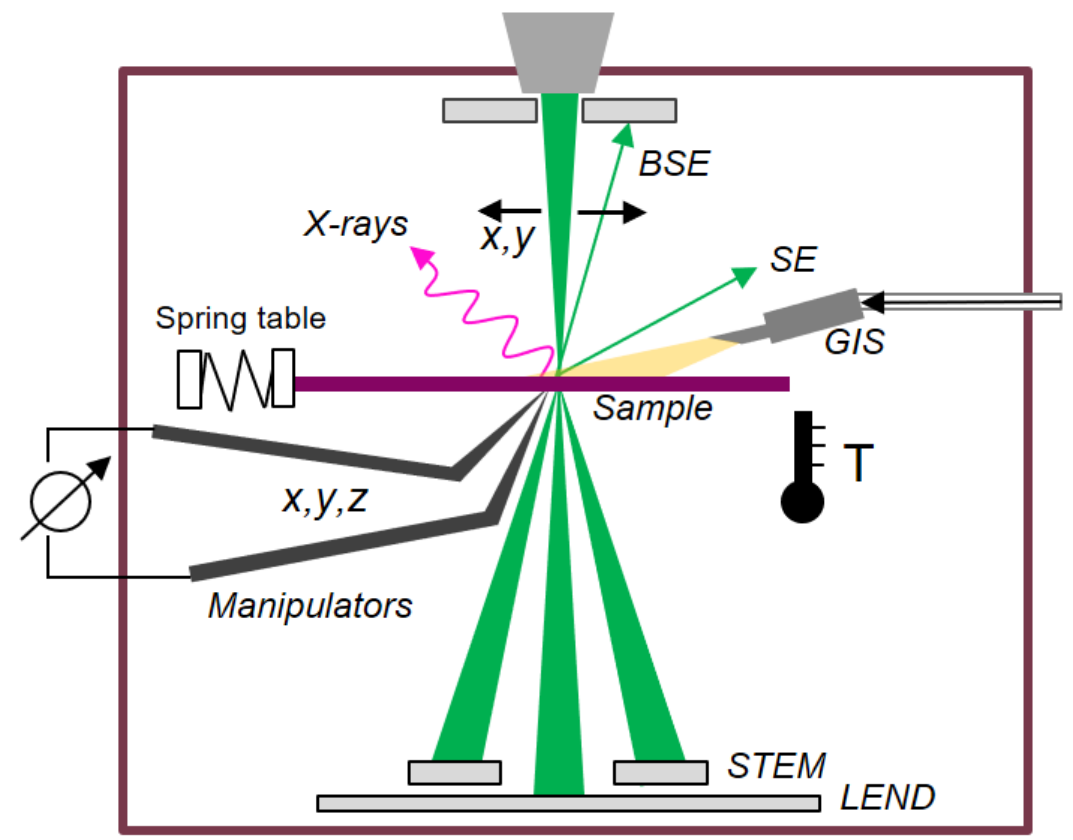

Figure 1. Versatile SEM setup combining STEM and LEND with various in situ tools including micro-manipulators, spring table for force measurement, gas injection system and heating device (schematic).

a)

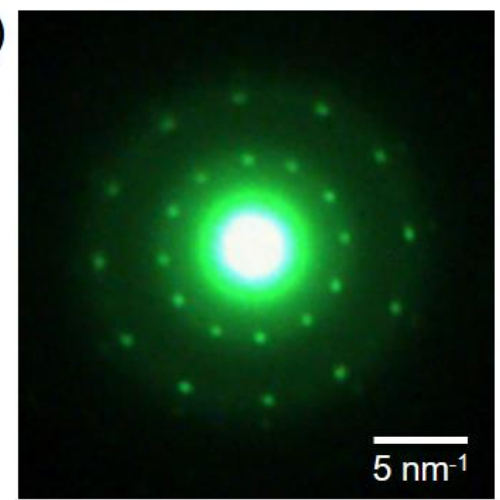

b)

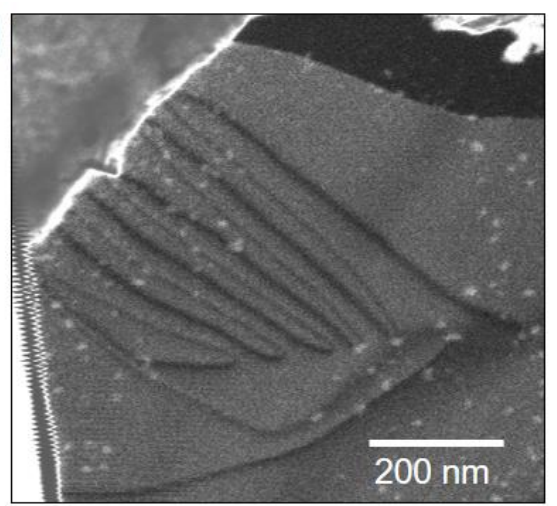

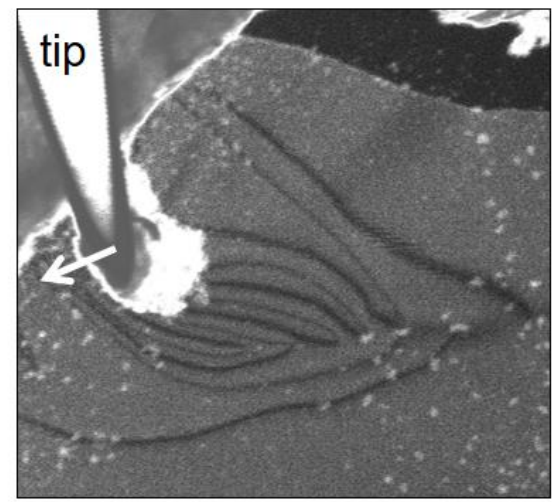

Figure 2. a) LEND pattern of rotated bilayer graphene taken with custom-built diffraction setup in SEM at $20 \mathrm{keV}$ primary electron energy. b) Dark-field STEM image in SEM showing diffraction contrast from basal plane dislocations in bilayer graphene. c) In situ mechanical manipulation of dislocations using a micro-manipulation with sharp tip. 